\section{Embarazo en hemodiálisis crónica: experiencia de un hospital universitario}

\author{
ÚRSULA FIEDLER Z. ${ }^{1}$, MA. EUGENIA SANHUEZA V. ${ }^{1}$, LUIS TORO C. ${ }^{1,2}$
}

\section{Pregnancy during chronic hemodialysis. A series of cases}

Background: Pregnancies in women with end stage renal failure are uncommon. However, correction of anemia and improvement in dialysis techniques increases the rate of successful pregnancies. Aim: To describe a 16 years' experience treating pregnant women on hemodialysis and to analyze maternal-fetal outcomes. Materials and Methods: Observational study of a dialysis center historical cohort in a university hospital, between 2001 and 2016. Results: Thirteen pregnancies were found in 11 women aged 23 to 32 years, 77\% on dialysis prior to pregnancy. Residual diuresis was 1,300 [625-1,575] $\mathrm{mL}$ in $24 \mathrm{hrs}$. The baseline hemoglobin was 9.0 [7.6-9.9] g/dL and 92\% of patients did not use contraception. The pre-dialysis blood urea nitrogen was 34 [29-36] mg /dL. An ultrasound to confirm pregnancy was done in all. At 23 [14-25] weeks of pregnancy, dialysis hours were increased, reaching 24 [19.5-24.0] hours per week. The most common complications were severe arterial hypertension (54\%), severe anemia (46\%), polyhydramnios (31\%) and severe intrauterine growth retardation (IUGR) (23\%). The median time of pregnancy at delivery was 34 [29-34] weeks. Neonatal median hospitalization length was 4 [4-32] days, with $18 \%$ of neonatal deaths. Conclusions: Pregnancies in dialysis are no longer exceptional. Despite better maternal and fetal outcomes, morbidity and mortality remains higher than in the normal population, which makes multidisciplinary management essential.

(Rev Med Chile 2019; 147: 709-717)

Key words: Kidney Failure, Chronic; Pregnancy; Renal Dialysis.
'Sección de Nefrología, Hospital Clínico Universidad de Chile. Santiago, Chile. ${ }^{2}$ Centro de Investigación Clínica Avanzada, Hospital Clínico Universidad de Chile. Santiago, Chile.

Trabajo no recibió financiamiento. Los autores declaran no tener conflictos de interés.

Recibido el 30 de octubre de 2018, aceptado el 14 de mayo de 2019.

Correspondencia a: Dra. Ursula Fiedler Zabra Santos Dumont 999, Independencia. Santiago, Chile. ufiedler@hcuch.cl
L os embarazos en pacientes en terapias de reemplazo renal distintas del trasplante se consideraban hasta años recientes como anecdóticos $^{1,2}$. La mayoría de las pacientes con enfermedad renal crónica terminal presentan ciclos anovulatorios y/o amenorrea, por lo que suelen considerarse como infértiles ${ }^{3}$. Además, las terapias dialíticas se han asociado con mayor riesgo de aborto, parto prematuro, muerte neonatal y complicaciones maternas, por lo que, el embarazo en estas condiciones significa un importante desafío médico. Sin embargo, junto a la introduc- ción de la eritropoyetina para la corrección de la anemia y la mejoría en las técnicas dialíticas, las gestaciones con éxito han aumentado en las últimas décadas ${ }^{3-5}$.

Por esta razón existe un incremento significativo de literatura acerca de casos exitosos de embarazos en diálisis, con el consiguiente establecimiento de algunos predictores de buenos resultados maternos y fetales, así como guías de manejo y parámetros de adecuación de diálisis que permitirían lograr estos objetivos ${ }^{3,6,7}$.

El objetivo del presente estudio es exponer la 
experiencia de un hospital nacional durante los últimos 16 años trabajando con embarazadas en diálisis crónica, así como evaluar, en la realidad local, el impacto que han tenido las intervenciones recomendadas en la literatura.

\section{Materiales y Métodos}

Se realizó un estudio observacional de cohorte histórica en la Central de Diálisis del Hospital Clínico de la Universidad de Chile, entre los años 2001 y 2016. Se obtuvo aprobación por parte del comité de ética institucional. Los criterios de inclusión fueron: pacientes de sexo femenino, mayores de 18 años, con enfermedad renal crónica avanzada, que presentaron al menos un embarazo que requirió terapia dialítica. Se excluyeron pacientes cuyo parto fue atendido en un centro de salud distinto del área del estudio.

Se recolectó la información demográfica de las pacientes, antecedentes gineco-obstétricos y nefrológicos, la modalidad y régimen dialítico previo al embarazo si es que lo recibían, tiempo de permanencia en diálisis previo al embarazo, parámetros de adecuación en diálisis, complicaciones maternas, características del parto y del recién nacido.

\section{Análisis estadístico}

Las variables categóricas se expresan como valor absoluto (porcentaje). Las variables continuas se expresan como mediana [percentil 25 - percentil 75]. Los análisis estadísticos se realizaron mediante los softwares Microsoft Excel v.15.0 y GraphPad Prism v.6.0.

\section{Resultados}

En un período de 16 años se encontraron 13 embarazos, en 11 mujeres distintas, del total de mujeres en terapia de reemplazo renal en nuestro centro. Ocho pacientes fueron derivadas al centro desde otras instituciones y 5 pacientes embarazadas eran usuarias crónicas de nuestra unidad. Con un total en la unidad de 92 mujeres en edad fértil, corresponde a una tasa de 5,4\% de embarazo en 16 años de mujeres usuarias crónicas de nuestra diálisis (tasa promedio de embarazo por año de $0,34 \%)$.
En la Tabla 1 se describen las características de las pacientes previas a la gestación.

La mediana de edad fue de 30 [23-32] años. Se logró precisar la causa de la enfermedad renal crónica en 92\% de las pacientes (12 casos): 6 con glomerulopatía crónica $(46,1 \%), 2$ pielonefritis crónica $(15,3 \%), 2$ nefropatía lúpica $(15,3 \%), 1$ glomerulonefritis focal y segmentaria $(7,6 \%)$ y 1 glomerulonefritis mesangiocapilar (7,6\%). El $76,9 \%$ de las pacientes se encontraban en diálisis previo al embarazo, con un tiempo de permanencia de 4 [3-28] meses y un esquema dialítico de $10,5[10,5-12,0]$ horas semanales. Sólo una de las pacientes se encontraba en peritoneodiálisis al momento de embarazo, cambiándose a hemodiálisis durante el segundo trimestre. La diuresis residual fue de 1.300 [625-1.575] $\mathrm{mL}$ en $24 \mathrm{~h}$. La hemoglobina previa al embarazo fue de $9[7,6-9,9] \mathrm{g} / \mathrm{dL}$.

En relación a los antecedentes gineco-obstétricos, $92 \%$ de las pacientes (12 casos) no usaban ningún método anticonceptivo, mientras que la paciente restante usaba método de barrera. En todas fue necesaria la realización de una ecografía como método de confirmación del embarazo, aunque en 53,8\% había ya un examen de gonadotrofina coriónica humana ( $\beta \mathrm{HCG}$ ) positiva en sangre u orina. El tiempo de embarazo al momento del diagnóstico fue de 10,5 [9,0-12,3] semanas y en $100 \%$ de las pacientes se trató de un embarazo no deseado.

\section{Adecuación en diálisis}

La totalidad de las pacientes se dializaron en máquinas Fresenius $4008 \mathrm{~S}$ y utilizaron filtros biocompatibles con membrana de polisulfona. En 92\% de los casos no se efectuó reuso de los filtros. En la Tabla 2 se presenta la edad gestacional a la que se incrementaron las horas de diálisis, el número de horas semanales en diálisis, los ajustes realizados para el manejo de la anemia y el nitrógeno ureico en sangre prediálisis.

De las tres pacientes que iniciaron diálisis durante el embarazo (23\%), el inicio de ésta fue a las 22 [15-24] semanas. El incremento en el número de horas de diálisis se efectuó a las 23 [14-25] semanas de gestación, alcanzando 24 [19,5-24] horas semanales. La terapia de reemplazo renal se realizó principalmente en forma ambulatoria (84\%), sin embargo, hubo 3 casos en los que se requirió hospitalizar transitoriamente por complicaciones, las que se describen en la Tabla 3. 


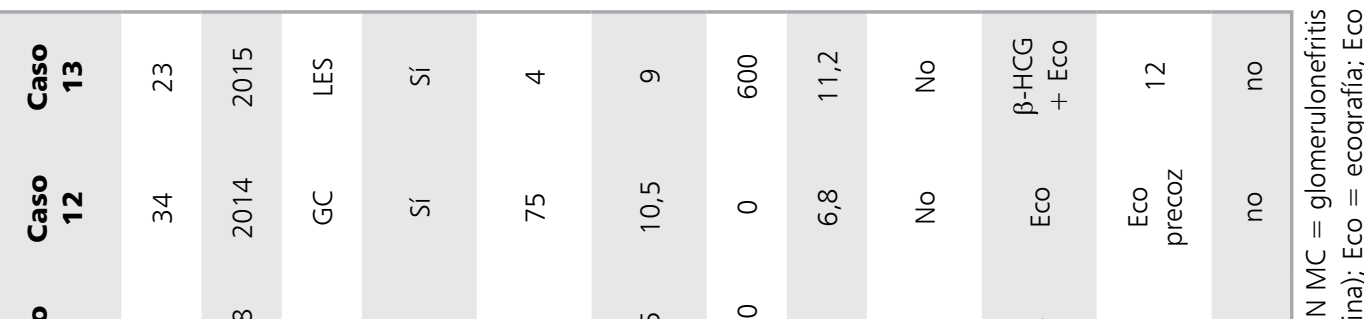

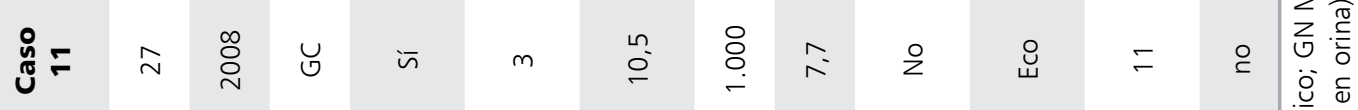

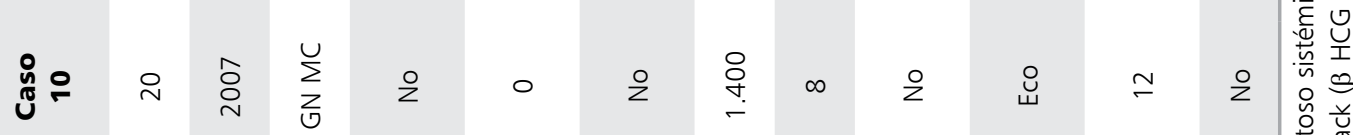

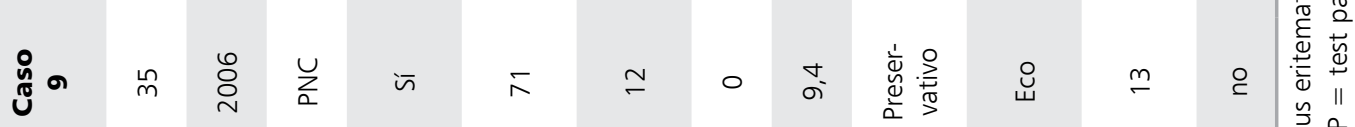

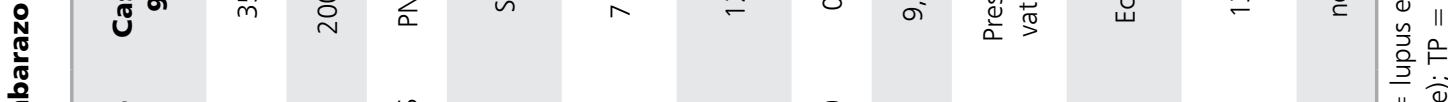

נூ

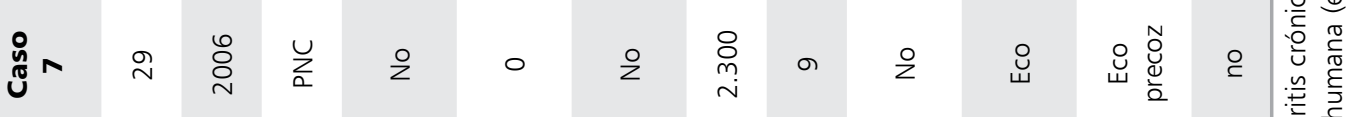

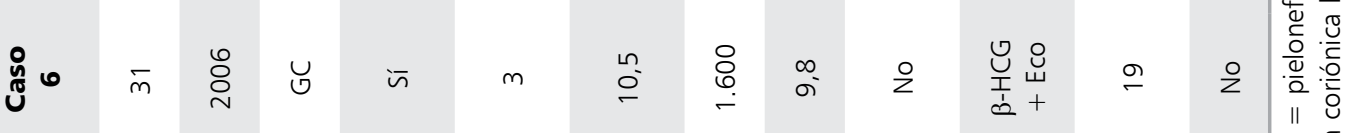

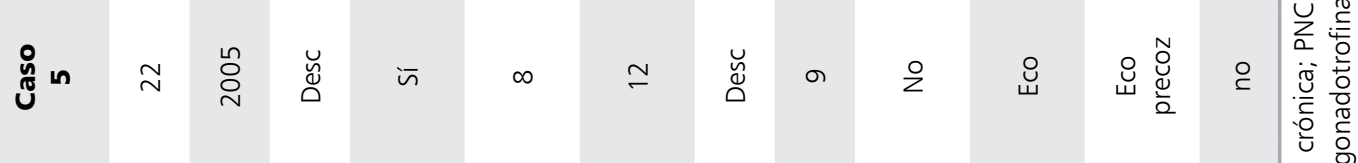

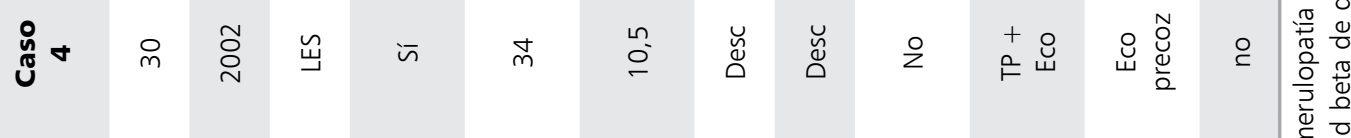

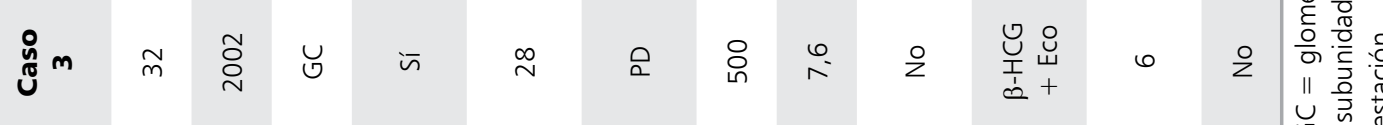

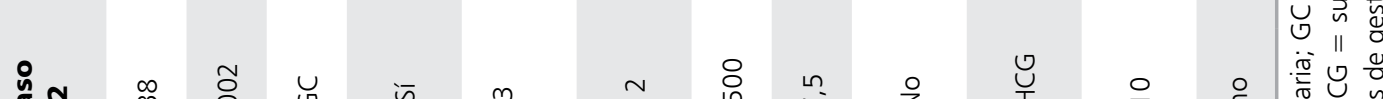

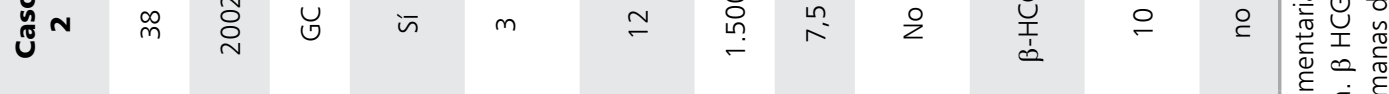

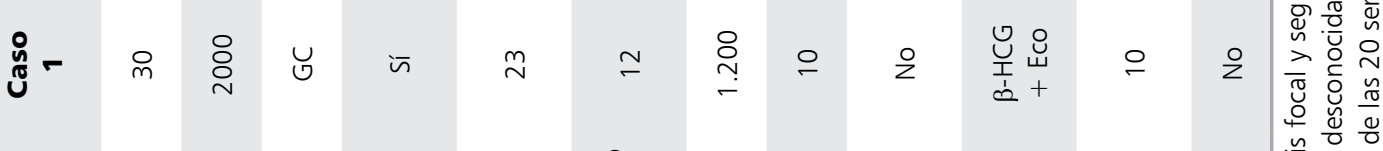

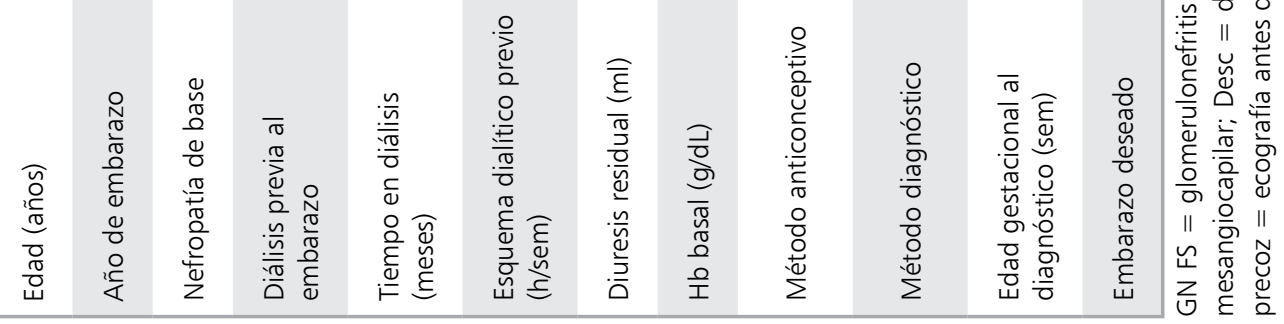




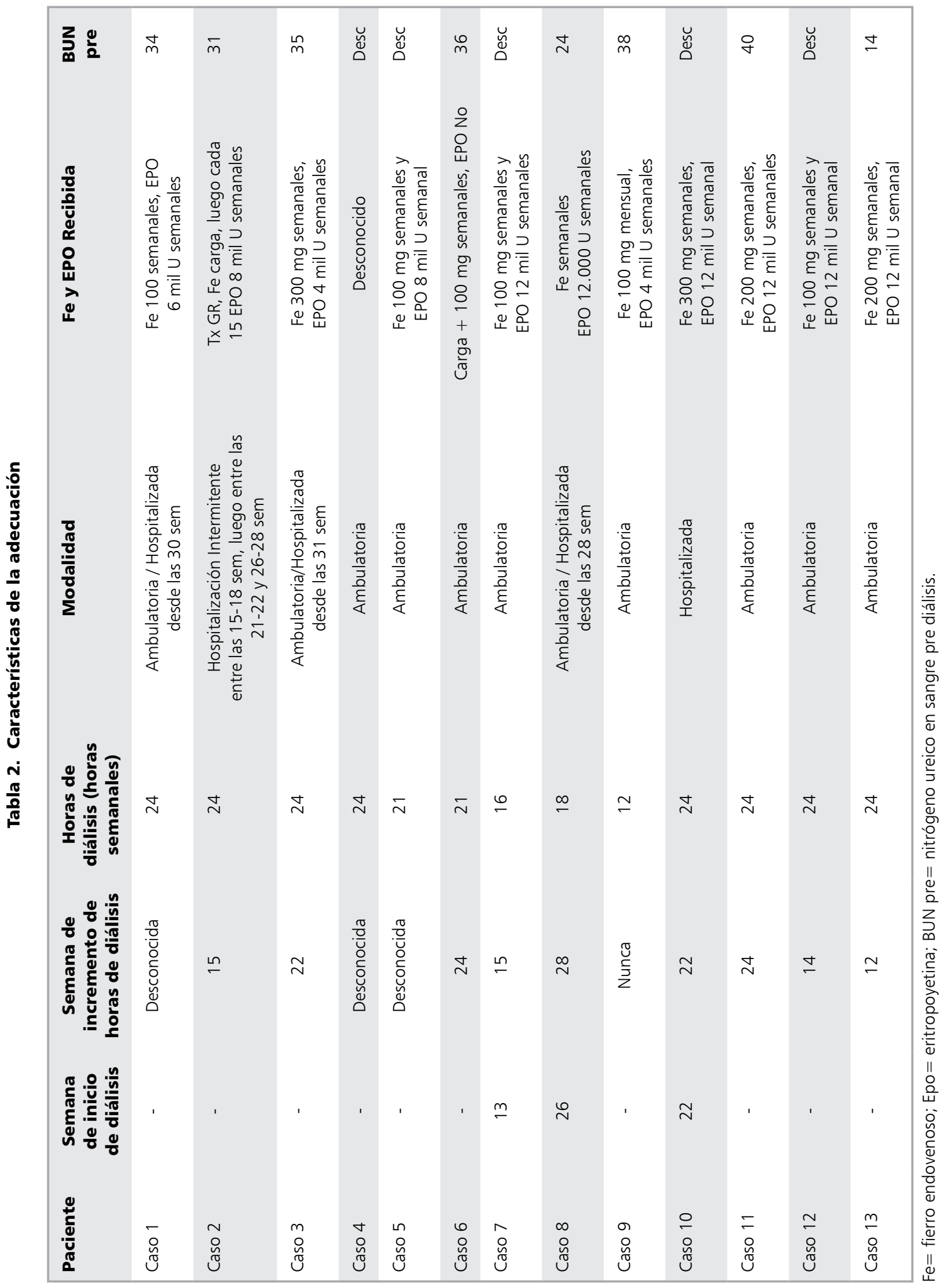




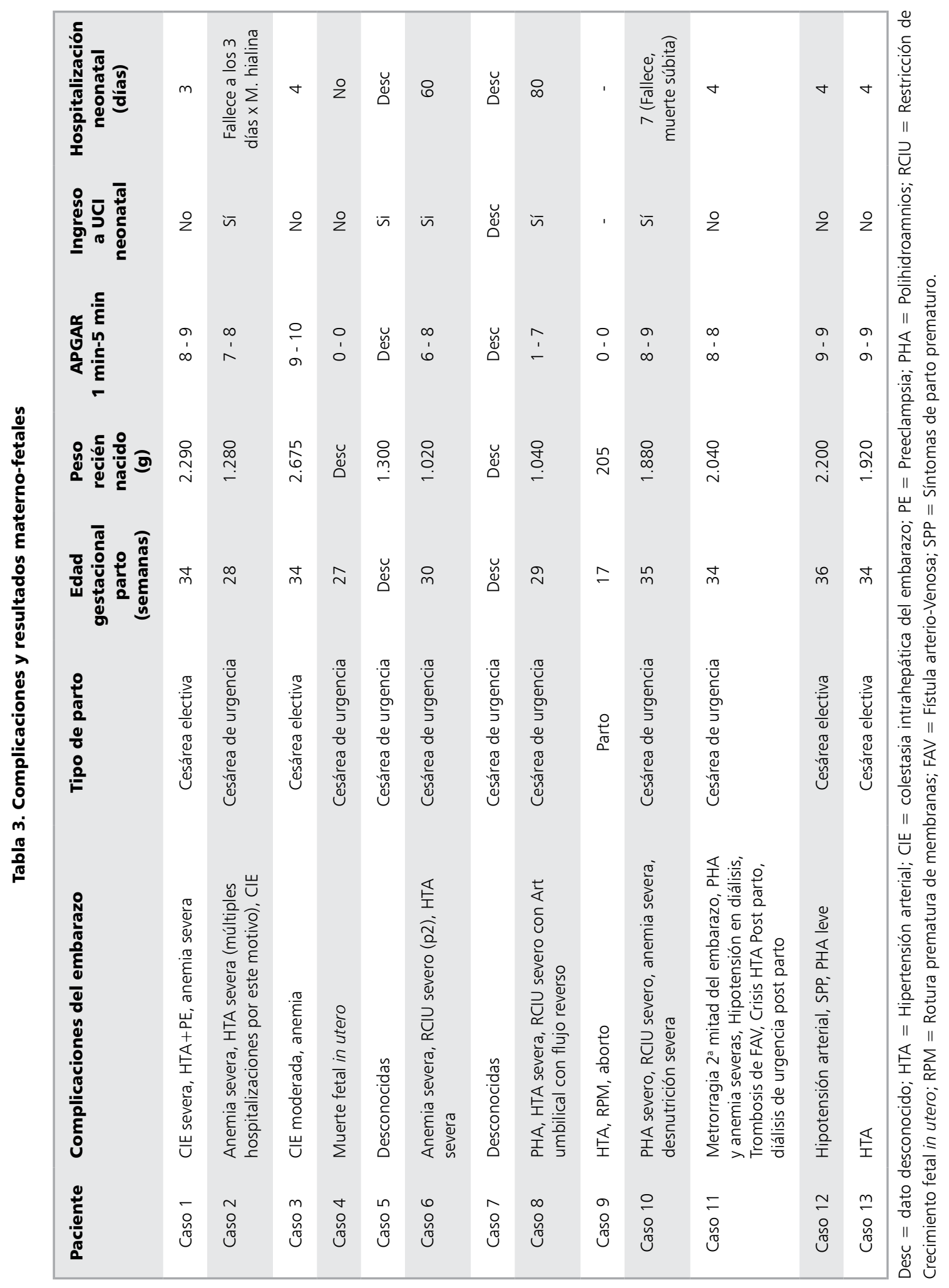


Respecto a los requerimientos de fierro endovenoso y eritropoyetina durante el embarazo, la mediana fue de 100 [100-200] mg semanales y 12.000 [6.000-12.000] U semanales respectivamente. El nitrógeno ureico prediálisis fue de 34 [29-36], sin embargo, este dato no fue consignado en 4 casos $(30 \%)$.

\section{Complicaciones y resultados perinatales}

Las complicaciones más frecuentes fueron la hipertensión arterial severa (54\%), anemia severa (46\%), polihidroamnios (31\%), restricción de crecimiento intrauterino (RCIU) severo (23\%), colestasia (23\%) y episodios de hipotensión severa asociada a las sesiones de hemodiálisis (15\%). Otras complicaciones menos frecuentes fueron preeclampsia, metrorragia, trombosis del acceso vascular, desnutrición severa, rotura prematura de membranas y aborto. Una paciente requirió diálisis de urgencia para manejo de crisis hipertensiva post parto.

En cuanto a la vía de resolución del embarazo, $100 \%$ fue mediante cesárea, $70 \%$ efectuadas de urgencia. La edad gestacional al momento del parto fue de 34 [29-34] semanas. El peso de los recién nacidos fue de 1.880 [1.040-2.040] g, con un puntaje de APGAR de 9 [8-9], al minuto 1 y 5 de nacidos. Hubo 2 abortos espontáneos (17 y 27 semanas). La mediana de hospitalización neonatal fue de 4 [4-7] días, con $18 \%$ de muertes neonatales ( 2 casos).

\section{Discusión}

En el presente trabajo se han descrito las características y resultados de 13 embarazos en mujeres con enfermedad renal crónica terminal que requirieron hemodiálisis. Sin embargo, el diagnóstico de embarazo en diálisis es difícil, incluso en las pacientes que aún tienen función renal residual, dado por lo inespecífico de los síntomas, los trastornos de los ciclos menstruales habituales en las pacientes con ERC avanzada y por la alteración de la eliminación de la $\beta$ HCG que en forma normal se produce vía renal ${ }^{3,8}$. Se necesita por lo tanto, un alto índice de sospecha, por ejemplo frente a anemia refractaria al uso de eritropoyetina, hiperemesis o hipotensión sin causa aparente. Todo esto conlleva a que el diagnóstico se haga en forma tardía, por lo cual desconocemos cual es la real tasa de embarazo en nuestras pacientes?.
En nuestra serie de casos la mayoría de las pacientes ya estaban en hemodiálisis crónica. Sólo una paciente se encontraba en terapia de reemplazo mediante diálisis peritoneal, la cual fue transferida a hemodiálisis durante el segundo trimestre de embarazo. Si bien en la literatura se reportan casos exitosos de embarazo en esta técnica, sigue siendo lo más frecuente que las pacientes sean ingresadas a hemodiálisis, por la mayor experiencia de los equipos con esta modalidad de terapia de reemplazo renal y para evitar las complicaciones derivadas del aumento de la presión intraperitoneal en el embarazo avanzado ${ }^{10,11}$.

Aunque la mayor parte de nuestras pacientes ya se encontraba en terapia de reemplazo renal antes de la concepción, la mediana de tiempo de permanencia previo a embarazarse fue de sólo 4 meses. Esto explicaría la alta diuresis residual encontrada en nuestras pacientes embarazadas, lo cual está descrito en la literatura que se correlaciona con mayores tasas de embarazo y mejor pronóstico materno y fetal ${ }^{12}$.

Llama la atención que más de $90 \%$ de las pacientes no utilizaban ningún método anticonceptivo, presentando en $100 \%$ de los casos embarazos no deseados/esperados. Esto debe hacernos reflexionar como tratantes de mujeres con ERC avanzada en edad fértil acerca de la posibilidad permanente de embarazo para su diagnóstico pre$\mathrm{COZ}$, así como realizar consejería y prevención $\mathrm{n}^{13-15}$.

Respecto al inicio de la diálisis durante el embarazo, ésta se hizo avanzado el $2^{\circ}$ trimestre, principalmente para optimizar el manejo nutricional de las pacientes. Asimismo el incremento en el número de horas de diálisis se efectuó con una mediana de 23 semanas de gestación, con una clara tendencia en los últimos años a iniciarla en forma precoz (alrededor de la semana 14), de acuerdo a las recomendaciones internacionales que se han relacionado con mejores resultados maternos y fetales ${ }^{1,16}$. Numerosos estudios han demostrado el beneficio de aumentar las horas semanales de diálisis, con un valor internacionalmente aceptado como óptimo de $24 \mathrm{~h}$ semanales, lo cual permite optimizar el manejo nutricional de las embarazadas, realizar ultrafiltraciones suaves para evitar la hipotensión y por ende la caída del flujo a la unidad fetoplacentaria, así como disminuir la exposición fetal a toxinas urémicas que conlleva al desarrollo de polihidroamnios. Todo lo anterior lleva permite prolongar el período gestacional, 
obteniendo mejor peso al nacer y mayor sobrevida neonatal ${ }^{2,16-19}$. Estas metas de diálisis intensiva fueron alcanzadas por nuestras pacientes. Trabajos posteriores, sin embargo, han mostrado mejores resultados en términos de sobrevida fetal, prematurez y complicaciones maternas con un mayor número de horas, no habiendo un estudio aún que revele resultados negativos a partir de un número de horas de hemodiálisis determinado ${ }^{20}$. En este mismo sentido, se recomienda buscar niveles de BUN prediálisis bajo $50 \mathrm{mg} / \mathrm{dL}$, lo cual se correlaciona con menos polihidroamnios, disminución del parto pretérmino y mejoría de los parámetros nutricionales en la embarazada en diálisis ${ }^{17,21}$. Esto fue logrado ampliamente por nuestras pacientes, pese a lo cual hubo 3 pacientes que igualmente cursaron con polihidroamnios.

A diferencia de otros trabajos, en este estudio no informamos los valores de Kt/V por sesión obtenidos de los exámenes mensuales tomados a las pacientes en diálisis, considerando que el $\mathrm{Kt} / \mathrm{V}$ tanto estándar como equilibrado calculado por fórmula de Daugirdas 2, sólo se encuentra validado para regímenes de diálisis trisemanal. Por lo tanto, si en futuros estudios quisiera utilizarse el $\mathrm{Kt} / \mathrm{V}$ como medida de dosis de diálisis debiéramos medir Kt/V semanal o el Kt, razón por la que las horas semanales de diálisis siguen siendo la meta de más fácil aplicación ${ }^{22}$.

La anemia severa fue una de las complicaciones más frecuentes encontradas y de difícil manejo, necesitándose dosis de fierro endovenoso y eritropoyetina durante el embarazo muy superiores a las indicadas habitualmente en diálisis en las pacientes no embarazadas, con la finalidad de mantener valores de hemoglobina sobre $10 \mathrm{~g} / \mathrm{dl}$ (la mediana fue de $100 \mathrm{mg}$ de hierro semanales y $12.000 \mathrm{U}$ de eritropoyetina semanales). La anemia en el embarazo se asocia a aumento de la incidencia de parto prematuro, de hecho Asimiya et al en un estudio en 24 pacientes embarazadas en hemodiálisis estableció correlación entre la hemoglobina materna y embarazo exitoso ${ }^{17}$. El uso de EPO además en el embarazo es seguro, no es teratogénico, ni tiene mayores repercusiones en la presión arterial. En esta misma línea se conoce que los suplementos orales de fierro en las embarazadas en diálisis son insuficientes y que el fierro endovenoso no tiene reportes de efectos adversos a nivel fetal, por lo que puede usarse según aumenten las demandas en el curso de la gestación ${ }^{17,23,24}$.
Otro de los problemas frecuentemente encontrados fue el difícil manejo de la presión arterial, con hipertensión arterial severa en más de la mitad de los casos. La mayoría de las pacientes se benefician de la diálisis intensiva y ajuste apropiado del peso seco para el manejo de este punto, requiriendo además ajuste de los medicamentos antihipertensivos a aquellos que no tengan efectos teratogénicos ${ }^{1,25}$. Es así como se pudo ver que en las pacientes manejadas con más horas de diálisis semanales no hubo casos de HTA severa ni necesidad de hemodiálisis de urgencia por crisis hipertensivas periparto. Sin embargo, la evaluación de este aspecto adquiere gran relevancia, debiéndose siempre intentar descartar una preeclampsia (PE) sobreagregada, la cual empeora los pronósticos maternos y fetales. El diagnóstico de una PE sobreagregada constituye un verdadero desafío clínico, pues algunas pacientes son anúricas y por lo tanto, no se puede objetivar proteinuria, otras en tanto tienen diuresis residual con proteinuria secundario a su enfermedad de base. Marcadores poco específicos como el ácido úrico y más recientemente biomarcadores derivados de factores angiogénicos y antiangiogénicos permiten seleccionar a grupos que tienen mayor riesgo de PE, así como predecir riesgo de complicación y de interrupción de embarazo, intentando de esta manera diferenciar aquellas hipertensiones gestacionales no complicadas de las que tienen PE sobreagregada. En este sentido, todas nuestras pacientes recibieron profilaxis de PE con aspirina $100 \mathrm{mg}$ desde fines del primer trimestre de embarazo, considerando que las mujeres con ERC avanzada son un grupo de alto riesgo para desarrollar $\mathrm{PE}^{26-29}$.

En cuanto a la vía de resolución del embarazo $100 \%$ fue mediante cesárea, valor superior lo observado en la literatura ${ }^{18,30}$. Setenta por ciento de ellas fueron efectuadas de urgencia, con una tendencia en los últimos años a la cesárea electiva. En ese mismo sentido la mediana de semanas de embarazo al momento del parto fue de 34 semanas, lo cual es superior a lo reportado internacionalmente en grupos de embarazadas similares, con valores cercanos a 32 semanas $^{6,31,33,34}$. El peso de los recién nacidos también ha ido en aumento, lo cual ha permitido tener resultados de APGAR mayores, menores tiempos de hospitalización neonatal y necesidad de UCI. Como consecuencia de todo esto, nuestra tasa de embarazos en diálisis 
exitosos es de 70\%, lo cual coincide con la literatura a la fecha ${ }^{32,33}$.

En conclusión, de nuestra revisión podemos afirmar que:

- Los embarazos en diálisis han dejado de ser excepcionales, teniendo en nuestro centro casi un caso por año.

- En los últimos casos hemos tenido mejores resultados maternos y fetales, pero aún con una morbimortalidad mayor a la población normal, lo cual hace imprescindible el manejo multidisciplinario.

- Los mejores resultados obtenidos son posiblemente consecuencia del mejor manejo de las cifras tensionales con la implementación de diálisis intensiva y de inicio precoz, así como de la experiencia ganada en conjunto por el equipo de nefrología y medicina materno fetal.

\section{Referencias}

1. Hou S. Pregnancy in chronic renal insufficiency and end stage renal disease. Am J Kidney Dis 1999; 33: 235.

2. Hou S. Pregnancy in women treated with dialysis: lessons from a large series over 20 years. Am J Kidney Dis 2010; 56 (1): 5-6.

3. Jungers P, Chauveau D. Pregnancy in renal disease. Kidney Int 1997; 52 (4): 871-85.

4. Schaefer RM, Kokot F, Wernze H, Geiger H, Heidland A. Improved sexual function in hemodialysis patients on recombinant erythropoietin: a posible role for prolactin. Clin Nephrol 1989; 31 (1): 1-5.

5. Fischer MJ, Lehnerz SD, Hebert JR, Parikh CR. Kidney disease is an independent risk factor for adverse fetal and maternal outcomes in pregnancy. Am J Kidney Dis 2004; 43: 415 .

6. Giatras I, Levy DP, Malone FD et al. Pregnancy during dialysis:case report and management guidelines. Nephrol Dial Transplant 1998; 13: 3266.

7. Hou S. Modification of dialysis regimens for pregnancy. Int J Artif Organs 2002; 25: 823.

8. Levy DP, Giatras I, Jungers P. Pregnancy and end stage renal disease past experience and new insights. Nephrol Dial Transplant 1998; 13 (12): 3005-7.

9. Espinoza F, Romeo R, Ursu M, Tapia A, Vukusich A. Embarazos exitosos en hemodiálisis crónica: experiencia de un único centro. Rev Med Chile 2013; 141: 1003-9.

10. Hou S. Pregnancy in women treated with peritoneal dialysis: viewpoint 1996. Perit Dial Int 1996; 16 (5): 442-3.

11. Manisco G, Potì' M, Maggiulli G, Di Tullio M, Losappio
V, Vernaglione L. Pregnancy in end-stage renal disease patients on dialysis: how to achieve a successful delivery. Clin Kidney J 2015; 8 (3): 293-9.

12. Jesudason S, Grace BS, McDonald SP. Pregnancy outcomes according to dialysis commencing before or after conception in women with ERSD. Clin J Am Soc Nephrol 2014; 9: 143.

13. Piccoli GB, Conijn A, Consiglio V. Pregnancy in dialysis patients: is the evidence strong enough to lead us to change our counseling policy? Clin J Am Soc Nephrol 2010; 5: 62 .

14. Tong A, Jesudason S, Craig J, Winkelmayer W. Perspectives on pregnancy in women with chronic kidney disease: systematic review of qualitative studies. Nephrol Dial Transplant 2015; 30: 652-61.

15. Groothoff J. Pregnancy during dialysis: still a challenge to get there, but worth the effort. Nephrol Dial Transplant 2015; 30: 1053-5.

16. Hou S. Modification of dialysis regimens for pregnancy. Int J Artif Organs 2002; 25: 823.

17. Asamiya Y, Otsubo S, Matsuda Y. The importance of low blood urea nitrogen levels in pregnant patients undergoing hemodialysis to optimize birth weight and gestational age. Kidney Int 2009; 75: 1217.

18. Bamberg C, Diekmann F, Haase M, Budde K, Hocher B, Halle H, et al. Pregnancy on intensified hemodialysis: fetal surveillance and perinatal outcome. Fetal Diagn Ther 2007; 22: 289-93.

19. Hou S. Pregnancy in dialysis patients: where do we go from here? Semin Dial 2003; 16: 376.

20. Hladunewich MA, Hou S, Odutayo A. Intensive hemodialysis associates with improved pregnancy outcomes: a Canadian and United States cohort comparison. J Am Soc Nephrol 2014; 25: 1103.

21. Galland R, Traeger J, Arkouche W, Delawari E, Fouque D. Short daily hemodialysis and nutritional status. Am J Kidney Dis 2001; 37: 95-8.

22. Maduell F, Arias M. Dosis de diálisis. En: Lorenzo V, López Gómez JM (Eds) Nefrología al Día. http:// www.revistanefrologia.com/es-monografias-nefrologia-dia-articulo-dosis-dialisis-36.

23. Holley JL, Reddy SS. PRegnancy in dialysis patients: a review of outcomes, complications and management. Semin Dial 2003; 1 6(5): 384-8.

24. Levy A, Fraser D, Katz M, Mazor M, Sheiner E. Maternal anemia during pregnancy is an independent risk factor for low birthweight and preterm delivery. Eur J Obstet Gynecol Reprod Biol 2005; 122 (2).

25. Reddy SS, Holley JL. Management of the pregnant chronic dialysis patient. Adv Chronic Kidney Disease 2007; 14 (2): 146-55. 
26. Magee L, Pels A, Helewa M, Rey E, von Dadelszen P. Diagnosis, evaluation, and management of the hypertensive disorders of pregnancy. Pregnancy Hypertension: An International Journal of Women's Cardiovascular Health 2014; 4: 105-45.

27. Steegers EA1, von Dadelszen P, Duvekot JJ, Pijnenborg R. Pre-eclampsia. Lancet 2010; 376: 631-44.

28. Levine RJ, Lam C, Qian C, Yu KF, Maynard SE, Sachs BP, et al. Soluble Endoglin and Other Circulating Antiangiogenic Factors in Preeclampsia. N Engl J Med 2006; 355: 992-1005.

29. Nevis IF, Reitsma A, Dominic A. Pregnancy outcomes in women with chronic kidney disease: a systematic review. Clin J Am Soc Nephrol 2011; 6: 2587.

30. Yang LY, Thia EWH, Tan LK. Obstetric outcomes in women with end-stage renal disease on chronic dialysis: a review. Obstetric Medicine 2010; 3 (2): 48-53.

31. Romao JE, Luders C, Kahhale S, Pascoal IJ, Abensur H, Sabbaga E. Pregnancy in women on chronic dialysis. A single center experience with 17 cases. Nephron 1998; 78 (4): 416-22.

32. Luders C, Castro MC, Titan SM, De Castro I, Elias RM, Abensur H. Obstetric outcome in pregnant women on long term dialysis: a case series. Am J Kidney Dis 2010; 56 (1): 36-85.

33. Eroglu D, Lembet A, Ozdemir FN, Ergin T, Kazanci F, Kuscu E. Pregnancy during hemodialysis: Perinatal outcome in our cases. Transplant Proc 2004; 36 (1): 53-5.

34. Toma H, Tanabe K, Tokumoto T, Kobayashi C, Yagisawa T. Pregnancy in women receiving renal dialysis or transplantation in Japan: a nationwide survey. Nephrol Dial Transplant 1999; 14 (6): 1511-6. 